\title{
Macrophage activation syndrome in a patient lately diagnosed with malaria
}

\author{
Montemaggi $A^{1}$, Trapani $S^{1,5}$, Lachina $L^{1}$, Galli $L^{2,5}$, Simonini $G^{3,5}$, Sieni $E^{4}$, Resti $M^{1}$
}

Department of Heath Sciences, ${ }^{1}$ Pediatric Unit, ${ }^{2}$ Infectious Disease Unit, ${ }^{3}$ Rheumatology Unit, ${ }^{4}$ Oncohematology Unit, Meyer Children's Hospital, Florence, Italy, ${ }^{5}$ University of Florence

Introduction: Macrophage activation syndrome (MAS) is a hyperinflammatory condition, potentially fatal, that can be triggered by various autoinflammatory diseases, infections and malignancies.

Report: A 13-year old boy presented with a 4-days history of fever and myalgia. Exams showed anemia, thrombocytopenia, leukopenia and raised CRP. Blood smear was consistent with microcytic anemia. On admission he underwent IVIG infusion and ceftriaxone. His clinical conditions deteriorated with unremitting fever, dyspnoea, hypotension and hepatosplenomegaly. Trilineage cytopenia, high ferritin, increased CRP, decreased ESR, hypofibrinogemia, hypertrigliceridemia and hypoalbuminemia were consistent with the diagnosis of Macophage Activation Syndrome (MAS)/ Hemophagocytic Lympho-Histiocitosis (HLH). Myeloaspirate showed excessive macrophage activity with hemophagocytosis and no blasts.

\begin{tabular}{|l|c|}
\hline 2004 Diagnostic criteria for HLH & $\begin{array}{c}\text { Index } \\
\text { case }\end{array}$ \\
\hline Clinical criteria & \\
\hline Fever & yes \\
\hline Splenomegaly & yes \\
\hline Laboratory criteria & \\
\hline Cytopenia (affecting 2 of 3 cell lines in peripheral blood) & yes \\
\hline Hemoglobin $<9$ g/dl & yes \\
\hline Platelet $<100 \times 10^{3}$ per dl & yes \\
\hline Neutrophil $<1,000$ per dl & yes \\
\hline Fibrinogen $<1.5$ g/L and/or & \\
\hline Hyper triglyceridemia (fasting triglyceride $>3.0$ mmol/l) & yes \\
\hline Histopathologic criteria & \\
\hline Hematophagocytosis in bone marrow or spleen or lymph nodes & yes \\
\hline No evidence of malignancy & yes \\
\hline Additional criteria & \\
\hline Low or absent NK activity & no \\
\hline Ferritin $>500$ g/L & yes \\
\hline Soluble CD 25 (i.e. IL2 receptor) 2,400 U/ml & no \\
\hline
\end{tabular}

Hemocultures, and PCR for bacteria and viruses on blood resulted negative. Genetic risk factors for MAS/fHLH were negative. He was started on high-dose corticosteroids and on etoposide and cyclosporine shortly after, due to poor response. Two doses of anakinra were also given for persisting fever. Due to the scarce improvement, other possible infectious MAS triggers were reconsidered: $P C R$ for malaria resulted positive and his peripheral blood smears confirmed forms $P$. falciparum (parasite index 1\%).
He was started on iv artesunato and quinine chlorydrate with subsequent clinical improvement.

Oral steroids and cyclosporine were gradually tapered in 30 days. He did not have history of recent travels out of Italy.

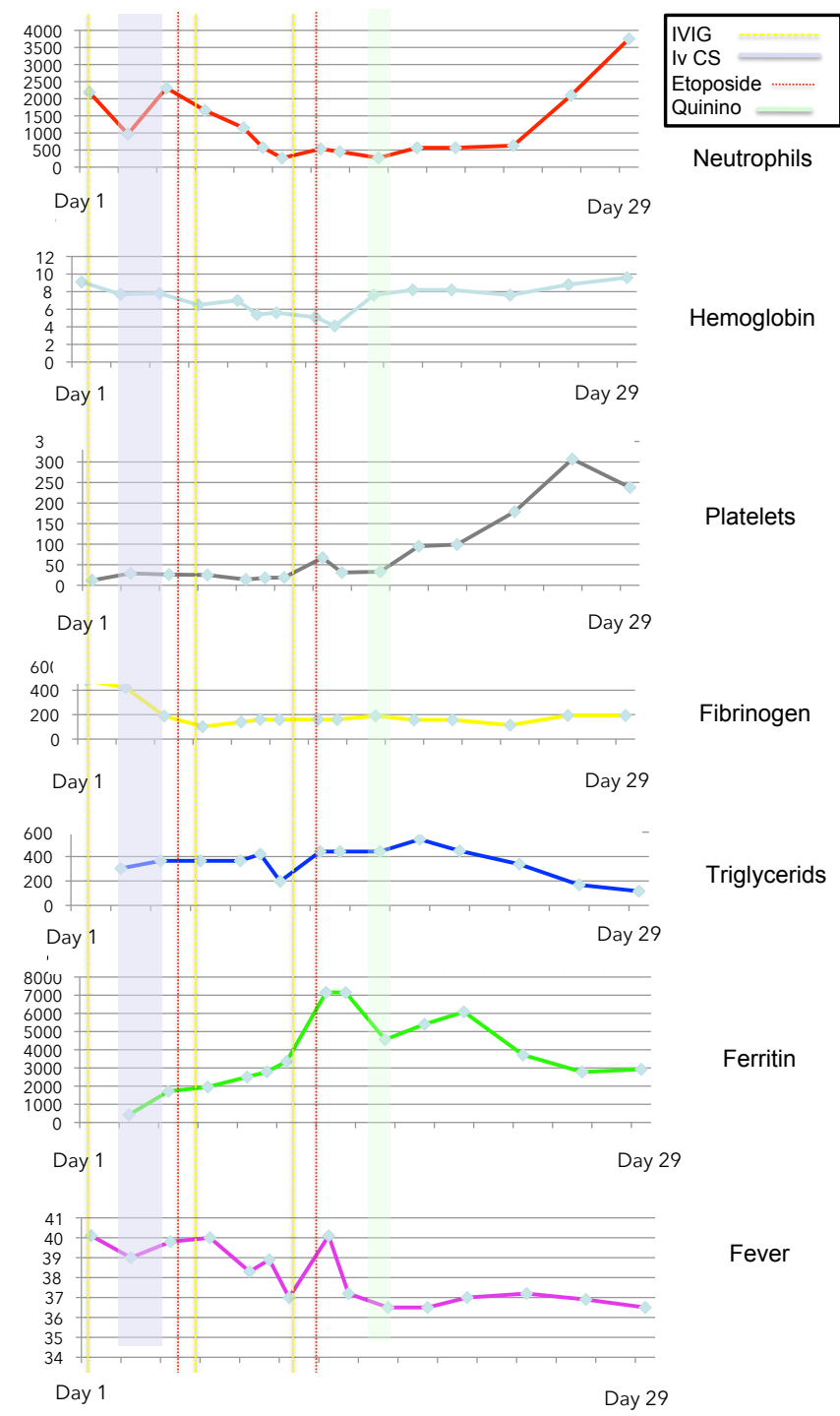

Conclusion: The association between MAS and malaria has been occasionally described. In our patient, malaria was diagnosed only after the failure of specific treatment for MAS. As many features of malaria and MAS can overlap, clinicians should be alert to investigate both conditions in cases with unremitting fever and pancytopenia. 